\title{
Biologia reprodutiva de Psychotria carthagenensis (Rubiaceae), espécie distílica de fragmento florestal de mata ciliar, Centro-Oeste do Brasil
}

\author{
Reproductive biology of Psychotria carthagenensis (Rubiaceae), \\ a distylous species of riparian forest fragments, West Central Brazil
}

Ana Kelly Koch ${ }^{1}$, Patrícia Campos da Silva ${ }^{1}$ \& Celice Alexandre Silva ${ }^{1,2}$

\begin{abstract}
Resumo
Este estudo teve por objetivos analisar a fenologia de floração, a biologia e a morfologia floral, o sistema e o sucesso reprodutivo dos morfos florais de Psychotria carthagenensis Jacq. O período de floração se estendeu de maio (estação seca) a janeiro (estação chuvosa). A floração dos morfos florais foi sincronizada e o número médio de inflorescências, de botões em pré-antese e de flores abertas por indivíduos foram semelhantes. A antese das flores de ambos os morfos foi de 12 horas. Houve diferença significativa no comprimento da corola, dos lobos estigmáticos, das anteras e altura do estilete entre os morfos florais. A viabilidade de pólen foi alta entre ambos os morfos. A formação de frutos ocorreu em polinizações intramorfos e intermorfos e não houve diferença significativa na produção de frutos e sementes em tratamentos intermorfos. Os atributos florais analisados sugerem que os dois morfos contribuem similarmente para a manutenção da espécie na área de estudo. Palavras-chave: fenologia de floração, heterostilia, morfologia floral, sucesso reprodutivo.
\end{abstract}

\begin{abstract}
This study aims to examine flowering phenology, floral biology and morphology, and the reproductive system and success of the floral morphs of Psychotria carthagenensis Jacq. The long flowering period lasts from May (dry season) to January (rainy season). Flowering of the floral morphs was synchronized and the number of inflorescences, buds in pre-anthesis and flowers per plant were similar. Anthesis of the flowers of both morphs was 12 hours. Significant differences were observed in the corolla length, the stigmatic lobes, anthers and styles between floral morphs. Pollen viability was high in both morphs. Intermorph and intramorph pollinations resulted in fruit set. There was no significant difference between intermorph treatments in fruit and seed production. The flower attributes indicate that both morphs contribute similarly to species persistence in the study area.

Key words: floral morphology,flowering phenology, heterostyly, reproductive success.
\end{abstract}

\section{Introdução}

Rubiaceae Juss. possui cerca de 630 gêneros e 10.700 espécies (Robbrecht 1988). O gênero Psychotria é o maior dessa família, com aproximadamente 1600 espécies (Almeida \& Alves 2000), comumente encontradas em sub-bosque de florestas tropicais e representadas por ervas, arbustos, pequenas árvores e raramente epífitas (Taylor 1996; Lopes \& Buzato 2005). As espécies desse gênero apresentam características florais como: antese diurna, corola tubular, coloração variando entre branca, creme ou amarela (Coelho \& Barbosa 2004; Teixeira \& Machado 2004a), e polinização por abelhas, moscas ou mariposas (Coelho \& Barbosa 2004) e beija-flores (Almeida \& Alves 2000).

A heterostilia é um polimorfismo floral controlado geneticamente, representado pela formação de dois (distilia) ou três (tristilia) tipos florais, sendo mais comum a ocorrência da distilia (Ganders 1979; Barrett 1992). Espécies distílicas são

'Universidade do Estado de Mato Grosso, Centro de Estudos, Pesquisa e Desenvolvimento Agro-Ambiental (CPEDA), Depto. Ciências Biológicas, Lab. Botânica, Rod. MT 358, km 07, C. P. 287, 78300-000, Tangará da Serra, MT, Brasil.

${ }^{2}$ Autor para correspondência: celicealexandre645@hotmail.com 
aquelas que apresentam posicionamento recíproco de estigmas e anteras entre dois morfos florais diferentes, caracterizado como "brevistilos" (B) e "longistilos" (L), pertencentes a indivíduos distintos. Além da distilia, espécies heterostílicas caracterizam-se por apresentarem mecanismos de incompatibilidade onde, geralmente, apenas os cruzamentos intermorfos resultam na formação de frutos (Barrett 1992). Em espécies distílicas o sucesso reprodutivo também depende de uma razão equilibrada (1:1) entre os morfos, denominada isopletia (Ganders 1979).

Estudos sobre a biologia reprodutiva de espécies de Psychotria em território brasileiro envolveram diferentes aspectos, tais como: fenologia de floração (Grandisoli 1997; Silva 2007), morfometria floral (Castro et al. 2004; Rossi et al. 2005), razão entre morfos (Castro et al. 2004; Silva 2007), sistema reprodutivo (Rossi et al. 2005; Pereira et al. 2006b; Silva 2007) e sucesso reprodutivo (Ramos \& Santos 2005; Silva 2007). No entanto, poucos estudos abordaram os aspectos reprodutivos de cada morfo floral.

Nesse sentido, o presente trabalho tem por objetivo ampliar os conhecimentos sobre a biologia reprodutiva de Psychotria carthagenensis Jacq. e assim auxiliar na compreensão dos mecanismos de manutenção da espécie no fragmento florestal estudado.

\section{Material e Métodos}

\section{Área de estudo}

O estudo foi realizado no período de maio de 2007 a março de 2008 em um fragmento florestal de mata ciliar (14³8'48,90”'S e 57²6’07,42”W) com cerca de 2 ha, no município de Tangará da Serra MT. O clima da região é tropical úmido megatérmico (AW), segundo a classificação de Köppen (1948) com temperatura média anual de $24,4^{\circ} \mathrm{C}$. A estação chuvosa estende-se de outubro a abril e a de seca de maio a setembro. A precipitação média anual é de $1.500 \mathrm{~mm}$ e a umidade relativa do ar de $70-80 \%$ (Vela et al. 2006). O solo é do tipo Latossolo Vermelho Disférrico de textura muito argilosa (Serigatto 2006). A fitofisionomia do local de estudo está associada a um curso d'água, caracterizado por árvores predominantemente eretas que variam em altura e não formam galerias, apresentando aspecto semidecíduo (Ribeiro \& Walter 1998).

\section{Espécie estudada}

Psychotria carthagenensis é um arbusto distílico de 1-3 m de altura que ocorre em vegetação ciliar inundável, caapão de vazante ou em solos arenosos ou argilosos (Pott \& Pott 1994). As folhas são glabras, simples, opostas, com estípulas interpeciolares inteiras e caducas. As inflorescências são cimeiras paniculiformes, terminais; flores apresentam corola campanulada, branca e denso anel de pêlos no interior do tubo corolar; ovário ínfero, bilocular com disco nectarífero na parte superior em forma de anel (Consolaro 2004; Pereira et al. 2006a). Os frutos são drupáceos, vermelho intenso na maturidade, ornitocóricos (Pott \& Pott 1994). Distribui-se nos Estados Unidos, México, América Central, América do Sul e Antilhas (Burger \& Taylor 1993). No Brasil, ocorre desde o estado do Amazonas até o do Rio Grande do Sul (Andersson 1992).

Espécimes testemunhos estão depositados no Herbário Central da Universidade Federal de Mato Grosso (UFMT 38.626).

\section{Fenologia de floração}

Para os estudos fenológicos foram etiquetados e identificados quanto ao morfo, todos os indivíduos em fase reprodutiva de Psychotria carthagenensis encontrados na área de estudo. Estes indivíduos $(\mathrm{N}=37)$ foram acompanhados semanalmente e, a cada dia de avaliação, foram registrados: o número de inflorescência, de botões e de flores abertas por indivíduo. Para se evitar a amostragem de indivíduos clonais, foram avaliados indivíduos com pelo menos 3 metros de distância entre si (Silva 2007).

\section{Biologia e morfometria floral}

Para o estudo da biologia floral foi observada a longevidade das flores, das 05:00 às 18:00 hs de 20 botões de cada morfo provenientes de quatro indivíduos diferentes, desde a abertura e exposição dos órgãos reprodutivos até a senescência, caracterizada pela perda de brilho e mudança na coloração da corola: de branca para creme.

A análise morfométrica foi realizada nos dois morfos florais. Para cada morfo foram coletadas cinco flores de cinco indivíduos diferentes, totalizando 25 flores por morfo floral. Nas flores foram mensurados, com auxílio de papel milimetradoe microscópio estereoscópico, os seguintes parâmetros: altura dos estames e estilete; comprimento das anteras, do tubo da corola, dos lobos da corola e dos lobos estigmáticos (Fig. 1). As medidas dos morfos foram comparadas entre si pelo teste $t$, com auxílio do Programa “General Linear Model”'(StatSoft 2002). 


\section{Viabilidade dos grãos de pólen}

Para a determinação da viabilidade dos grãos de pólen, foram coletados cinco botões em préantese de cinco indivíduos diferentes para cada morfo, totalizando 25 botões por morfo floral. Estes botões foram estocados em ácido acético e, posteriormente, todas as anteras de cada botão foram maceradas em lâminas, sobre uma gota de carmim acético (Radford et al. 1974). Em cada lâmina foram contados até 200 grãos de pólen (Silva 2007).

\section{Sistema reprodutivo}

Para realizar os tratamentos de polinização manual utilizou-se a metodologia de Bawa \& Beach (1983): B × B e L × L (autopolinização); B × B e L × L (polinização intramorfos); $\mathrm{B} \times \mathrm{Le} \mathrm{L} \times \mathrm{B}$ (polinização intermorfos). Para isso, inflorescências jovens ( $\mathrm{N}=$ $148 \mathrm{~B} \mathrm{e} \mathrm{N}=178 \mathrm{~L}$ ) foram isoladas com sacos de tecido tipo "organza" e as flores polinizadas $(\mathrm{N}=249 \mathrm{~B}$ e $\mathrm{N}=253 \mathrm{~L}$ ) tiveram seus pedicelos marcados com linhas coloridas (cada cor representando um tipo de polinização). As polinizações manuais foram realizadas no período da manhã (entre 7:00 e 9:00 h). Após os tratamentos, as flores permaneceram ensacadas até a queda das flores ou frutificação. A maioria das flores ( $\mathrm{N}=214 \mathrm{~B}$ e $235 \mathrm{~L}$ ) tiveram seus pedicelos marcados como controle, para avaliar a eficiência da polinização natural.

\section{Sucesso reprodutivo}

O sucesso reprodutivo, aqui definido pela formação de frutos e sementes oriundos de polinizações naturais (sensu Dafni 1994), foi verificado no ano de 2008 em cinco indivíduos de cada morfo. Em cada indivíduo amostrado foram coletados, aleatoriamente, até cinco inflorescências com frutos totalmente desenvolvidos. Realizou-se a contagem do número de frutos por inflorescência e o número de sementes (uma ou duas) por fruto, segundo Silva (2007). A estimativa do sucesso reprodutivo (produção total de sementes por planta) foi obtida pela multiplicação do número médio de sementes por fruto pelo número médio de frutos por morfo.

\section{Resultados e Discussão}

\section{Fenologia de floração}

Psychotria carthagenensis floresceu de maio/ 2007 (início da estação seca), estendendo-se até janeiro/2008 (estação chuvosa). Houve sobreposição no período de floração de ambos os morfos, o pico de floração do morfo brevistilo antecedeu ao do morfo longistilo em um mês (Fig. 2). Não houve diferença significativa no número de inflorescências por individuo entre os morfos florais $(\mathrm{N}=148 \mathrm{~B}$ e $178 \mathrm{~L}$; $\mathrm{F}=0,0035 ; \mathrm{p}=0,95)$, no número de botões em pré antese por indivíduo $(\mathrm{N}=2231 \mathrm{~B}$ e $3053 \mathrm{~L} ; \mathrm{F}=0,088$; $\mathrm{p}=0,77)$ e no número de flores abertas por indivíduo $(\mathrm{N}=1999$ B e $2620 \mathrm{~L} ; \mathrm{F}=0,046 ; \mathrm{p}=0,83)$ (Tab. 1).

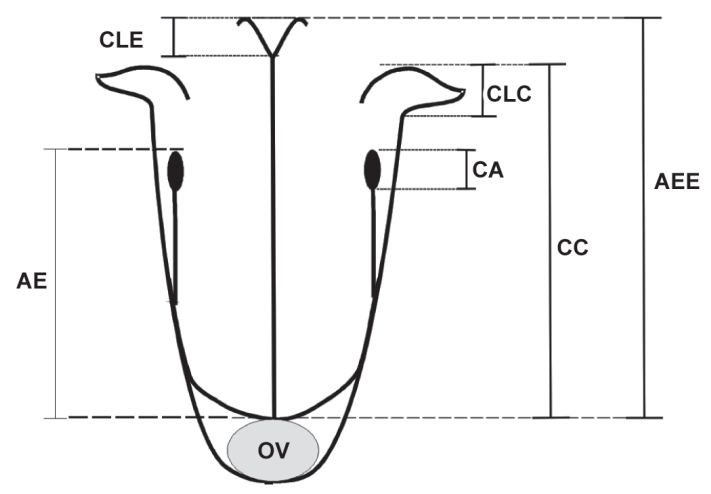

Figura 1 - Parâmetros para medidas florais de Psychotria carthagenensis no morfo longistilo. CC: comprimento da corola; CLC: comprimento dos lobos da corola; AEE: Altura do estilete com estigma; CLE: comprimento dos lobos estigmáticos; $\mathrm{CA}$ : comprimento das anteras; $\mathrm{AE}$ : altura do estame; OV: ovário ínfero. Figure 1 - Parameters to floral measurements of Psychotria carthagenensis in the pin morph. CC: length of the corolla; CLC: length the lobes of the corolla; AEE: height of style with stigma; CLE: length of the stigma lobes; CA: length of the anthers; AE: height of the stames; OV:infero ovary.

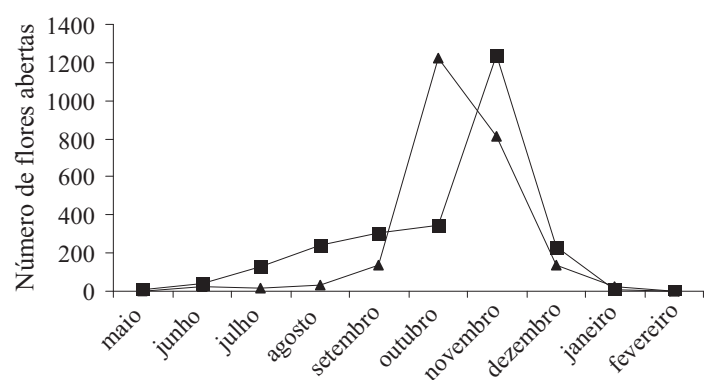

Figura 2 - Período de floração de Psychotria carthagenensis, em fragmento florestal de mata ciliar em Tangará da Serra-MT: (A) morfo brevistilo, ( morfo longistilo.

Figure 2 - Flowering period of the Psychotria carthagenesis, in a riparian forest fragment in Tangará da Serra-MT: (A) thrum morph, (ם) pin morph. 
Tabela 1 - Médias e desvio padrão de inflorescências, botões florais em pré antese e flores abertas/individuo em Psychotria carthagenensis em fragmento mata ciliar, Tangará da Serra-MT.

Table 1 - Averages and standard deviation of the inflorescences, pre-anthesis buds and open flowers/individual in Psychotria carthagenesis in a riparian forest fragment in Tangará da Serra-MT.

\begin{tabular}{lccc}
\hline $\begin{array}{l}\text { Morfo } \\
\left(\mathbf{N}^{\mathbf{0}} \text { indivíduos }\right)\end{array}$ & $\begin{array}{c}\text { Inflorescências/Indivíduo } \\
\bar{X} \pm \mathbf{D P}\end{array}$ & $\begin{array}{c}\text { Botões em } \text { pré-antese/Indivíduo } \\
\bar{X} \pm \mathbf{D P}\end{array}$ & $\begin{array}{c}\text { Flores abertas/Indivíduo } \\
\bar{X} \pm \mathrm{DP}\end{array}$ \\
\hline Brevistilo (17) & $8,70 \pm 7,70$ & $131,23 \pm 126,90$ & $117,58 \pm 93,23$ \\
Longistilo (20) & $8,9 \pm 11,52$ & $152,65 \pm 273,72$ & $131,00 \pm 236,40$ \\
\hline
\end{tabular}

A floração de Psychotria carthagenensis, restrita a alguns meses do ano, é do tipo "anual" segundo (Newstron et al. 1994), semelhante ao observado em P. nuda (Cham. \& Schltdl.) Wawra, $P$. brasiliensis Vell. (Almeida \& Alves 2000), Palicourea macrobotrys Roem. \& Schult. (Coelho \& Barbosa 2003), P. barbiflora DC. (Texeira \& Machado 2004a), P. carthagenensis (Consolaro 2004), P. sessilis Vell., P. hastisepala Müll. Arg. e P. conjungens Müll. (Silva 2007). Espécies com floração "extensiva", caracterizada por uma produção diária de flores por um período de até sete meses (Bawa \& Beach 1983), tal como observado em $P$. carthagenensis, tendem a maximizar o florescimento em períodos favoráveis a polinizadores e reprodução (Grandisoli 1997).

A sincronia de floração entre os morfos florais indica que ambos podem contribuir de maneira semelhante para o sucesso reprodutivo de $P$. carthagenensis, favorecido por polinizações intermorfos. Sincronia de floração entre morfos foi observada também em outras espécies de Psychotria, tais como: P. poeppigiana Müll. Arg. (Coelho \& Barbosa 2004), P. suterella Müll. Arg. (Lopes \& Buzato 2005), P. sessilis, P. hastisepala e P.conjugens (Silva 2007).

Foram identificados 20 indivíduos do morfo longistilo e 17 brevistilos de $P$. carthagenensis na área de estudo, ou seja, a população estudada está muito próxima da distribuição equilibrada (1:1), caracterizando-a como isoplética. A isopletia é uma condição desejável para espécies heterostílicas, pois o equilíbrio entre os morfos florais em uma população é indicativo que os indivíduos de cada morfo podem contribuir similarmente para a manutenção da espécie (Murcia 1996). Isopletia também foi registrada em populações naturais de Psychotria barbiflora (Texeira \& Machado 2004a), P. poeppigiana (Coelho \& Barbosa 2004), P. sessilis, P. hastisepala e P.conjugens (Silva 2007) e Palicourea crocea (Sw.) Roem.\& Schult. (Mendonça \& Anjos 2006).
Porém, anisopletia (desvios na proporção de 1:1) foi registrada em Psychotria barbiflora (Monteiro et al. 1991) e P. carthagenensis (Consolaro 2004). Segundo Pereira et al. (2006b) a quebra do equilíbrio entre morfos pode ser resultante de populações recém estabelecidas ou que estão sob efeitos da fragmentação florestal e carecem de estudos mais aprofundados para o esclarecimento dessa questão.

\section{Biologia e morfometria floral}

A antese das flores de $P$. carthagenensis compreende cerca de $12 \mathrm{~h}$. A abertura floral ocorre nas primeiras horas da manhã, entre 05:00 e 06:00 h, em ambos os morfos, período em que os lobos da corola da maioria das flores já se encontravam totalmente separados e posicionados perpendicularmente em relação ao eixo floral, exibindo as estruturas reprodutivas. A senescência se deu entre 17:00 e 18:00 h do mesmo dia, quando a corola e o estilete apresentavam-se opacos e murchos. Foi observada em algumas flores a queda da corola antes que as mesmas apresentassem estado de senescência. A biologia floral verificada em $P$. carthagenensis confirma os dados obtidos por Consolaro (2004) para esta mesma espécie em mata de galeria do Triângulo Mineiro - MG, onde a antese diurna e longevidade floral de $12 \mathrm{~h}$ foram relacionadas ao período das atividades dos visitantes florais.

Segundo Dulberger (1992) flores heterostilicas têm longevidade curta, durando geralmente de um a dois dias. A antese diurna tem predominado entre as espécies do gênero Psychotria, tais como $P$. barbiflora (Teixeira \& Machado 2004a), P. poeppigiana (Coelho $\&$ Barbosa 2004) e P. ipecacuanha (Brot.) Stokes (Rossi et al. 2005) e observada também em outros gêneros da família Rubiaceae, como Manettia sp. (Passos \& Sazima 1995; Consolaro et al. 2005), Rudgea sp. (Castro \& Oliveira 2002), Palicourea sp. (Coelho \& Barbosa 2003; Mendonça \& Anjos 2006), Sabicea sp. (Teixeira \& Machado 2004b), entre outros. Essa similaridade no período de antese entre os gêneros 
Tabela 2 - Morfometria das flores de cada morfo brevistilo e longistilo de Psychotria carthagenensis, em fragmento florestal de mata ciliar, Tangará da Serra-MT.

Table 2 - Morphometry of flowers each thrum and pin morph of the Psychotria carthagenesis in a riparian forest fragment in Tangará da Serra-MT.

\begin{tabular}{lllllll}
\hline \multirow{2}{*}{ Medidas (comprimento-mm) } & \multicolumn{7}{c}{ Morfos } & \multicolumn{5}{c}{ Longistilo } \\
\hline & \multicolumn{2}{l}{ Brevistilo } & \multicolumn{5}{c}{} \\
Corola & $\bar{X}$ & DP & $\bar{X}$ & DP & T & P \\
Lobos da corola & $6,68 \pm$ & 1,02 & $6,04 \pm$ & 0,84 & 2,40 & 0,01 \\
Lobos estigmáticos & $2,08 \pm$ & 0,27 & $2,12 \pm$ & 0,33 & $-0,46$ & 0,64 \\
Anteras & $1,80 \pm$ & 0,40 & $1,00 \pm$ & 0,00 & 9,79 & 0,001 \\
& $1,20 \pm$ & 0,40 & $1,00 \pm$ & 0,00 & 2,44 & 0,018 \\
Alturas/Estames & & & & & & \\
Estilete & $3,31 \pm$ & 0,04 & $2,14 \pm$ & 0,04 & 2,44 & 0,018 \\
& $3,92 \pm$ & 0,81 & $6,2 \pm$ & 0,76 & $-10,22$ & 0,001 \\
\hline
\end{tabular}

e espécies da família Rubiaceae sugere uma similaridade nos grupos de polinizadores com hábitos diurnos, variando em abelhas, moscas, mariposas (Coelho \& Barbosa 2004), borboletas e beija-flores (Passos \& Sazima 1995; Teixeira \& Machado 2004b; Consolaro et al. 2005).

Com relação às análises morfométricas em P. carthagenensis (Tab. 2), houve diferença significativa entre os morfos, no comprimento da corola $(p=0,01)$, dos lobos estigmáticos $(p=0,001)$ e das anteras $(\mathrm{p}=0,018)$ das flores brevistilas, quando comparadas às longistilas. O morfo longistilo apresentou diferença significativa $(p=0,001)$ na altura do estilete. Dimorfismos dos lobos estigmáticos e da corola têm sido registrados para outras espécies de Psychotria como P. nuda (Castro \& Araujo 2004; Pereira et al. 2006b), P. jasminoides Standl., P. birotula L.B.Sm $\&$ Downs, P. mapuorioides DC. e P. pubigera Blume ex Valeton (Castro et al. 2004); P. conjugens, $P$. hastisepala, $P$, hygrophiloides Benth. e $P$ sessilis Müll. Arg. (Pereira et al. 2006b). Segundo Dulberger (1992) pistilos brevistilos, por apresentarem lobos estigmáticos maiores, recebem maior quantidade de pólen que estigmas longistilos. Esse dimorfismo pode acarretar em um maior sucesso reprodutivo para o morfo brevistilo(Silva 2007). Entretanto, isso não parece ocorrer com $P$. carthagenensis, dado à semelhança no sucesso reprodutivo entre os morfos florais.

Diferenças significativas no comprimento das anteras também foram registradas em Psychotria nuda (Castro \& Araujo 2004; Pereira et al. 2006b), $P$. poeppigiana (Coelho \& Barbosa 2004) e em três populações de $P$. ipecacuanha (Rossi et al. 2005) e ainda em Palicourea longepedunculata (Pereira et al. 2006b). Dimorfismos das anteras têm sido relacionados com o tamanho de grãos de pólen, que são geralmente maiores no morfo brevistilo (Teixeira \& Machado 2004a; Rossi et al. 2005).

Viabilidade dos grãos de pólen

A viabilidade polínica foi semelhante entre os dois morfos florais $(\bar{x}=89,44 \pm 4,63 \mathrm{~B}$ e $\bar{x}=$ $91,68 \pm 5,94 \mathrm{~L} ; \mathrm{F}=2,06 ; \mathrm{p}=0,14)$. Alta viabilidade dos grãos de pólen, tal como verificada em $P$. carthagenensis, é uma característica comum em espécies heterostílicas (Dulberger 1992), registrada também em $P$. poeppigiana (Coelho \& Barbosa 2004), P. barbiflora (Teixeira \& Machado 2004a), P. sessilis, P.conjugens e P. hastisepla (Silva 2007), confirmando a participação de ambos os morfos como doadores de pólen na espécie.

\section{Sistema reprodutivo}

Os testes de polinizações manuais (Tab. 3) revelaram que a frutificação ocorreu principalmente após as polinizações intermorfos $(\mathrm{B} \times \mathrm{L}$ e $\mathrm{L} \times \mathrm{B})$. Uma pequena porcentagem de frutos foi registrado após polinizações intramorfos $(\mathrm{B} \times \mathrm{B}$ e $\mathrm{L} \times \mathrm{L})(\mathrm{Tab} .3)$. Embora o número de frutos formados pela polinização manual intermorfos tenha sido maior do que o encontrado no controle, não houve diferença significativa entre eles $(T=-4,43 ; \mathrm{p}=0,07)$. Os testes do controle indicam que os polinizadores têm realizado fluxo gênico satisfatório entre os morfos florais na natureza.

Os resultados obtidos através das polinizações intermorfos confirmaram o sistema de incompatibilidade esperado em espécies distilícas de Psychotria: $P$. barbiflora (Texeira \& Machado 2004a), P. suterella 
Tabela 3 - Polinização manual em flores de Psychotria carthagenensis, em fragmento florestal de mata ciliar, Tangará da Serra-MT.

Table 3 - Hand pollination in the flowers of Psychotria carthagenesis in a riparian forest fragment in Tangará da Serra-MT.

\begin{tabular}{lllll}
\hline Morfos*/Tratamentos & $\begin{array}{l}\text { Indivíduos } \\
\mathbf{N}^{\mathbf{0}}\end{array}$ & $\begin{array}{l}\text { Flores } \\
\mathbf{N}^{\mathbf{0}}\end{array}$ & \multicolumn{2}{l}{$\begin{array}{l}\text { Frutos } \\
\mathbf{N}^{\mathbf{0}}(\boldsymbol{\%})\end{array}$} \\
\hline $\mathrm{B} \times \mathrm{B}$ (autopolinização) & 3 & 65 & 0 & $(0,00)$ \\
$\mathrm{B} \times \mathrm{B}$ (intramorfo) & 3 & 33 & 3 & $(9,09)$ \\
$\mathrm{L} \times \mathrm{L}$ (autopolinização) & 3 & 72 & 0 & $(0,00)$ \\
$\mathrm{L} \times \mathrm{L}$ (intramorfo) & 3 & 55 & 2 & $(3,63)$ \\
$\mathrm{B} \times \mathrm{L}$ (intermorfo) & 3 & 126 & 54 & $(42,85)$ \\
$\mathrm{L} \times \mathrm{B}$ (intermofo) & 3 & 151 & 101 & $(66,88)$ \\
$\mathrm{C}$ ontrole & & & & $(34,11)$ \\
$\mathrm{B}$ & 2 & 214 & 73 & $(48,08)$ \\
L & 2 & 235 & 113 &
\end{tabular}

(Lopes \& Buzato 2005), P. sessilis, P. hastisepala e P. conjugens (Silva 2007). No entanto a autoincompatibilidade pode ser incompleta (pseudocompatibilidade sensu Lloyd \& Schoen 1992), tal como observada em $P$. carthagenensis e pode levar a formação de sementes após tratamentos de autopolinização. Na ausência do pólen oriundo de polinização cruzada, as espécies parcialmente autoincompatíveis têm baixo desempenho do auto pólen, o que resulta em uma baixa formação de sementes em flores autopolinizadas quando comparadas às flores de polinização cruzada (Lloyd \& Schoen 1992).

Quebra parcial da incompatibilidade foi observado em Palicourea macrobotrys (Coelho \& Barbosa 2003), Psychotria poeppigiana (Coelho \& Barbosa 2004), P. carthagenensis (Consolaro 2005) e $P$. ipecacuanha (Rossi et al 2005), nas quais foram observadas a formação de frutos após autopolinizações e polinizações intramorfos. Das espécies acima citadas, apenas $P$. poeppigiana apresentou os dois morfos florais na população ou "cluster" estudada, sugerindo que a quebra da incompatibilidade não é rigorosa e pode ser resultado de pressões diversas (Grandisoli 1997).

\section{Sucesso reprodutivo}

Não houve diferença significativa na produção de frutos (Tab. 4; $F=3,64 ; p=0,06$ ), na porcentagem de frutos com uma $(\mathrm{F}=3,42 ; \mathrm{p}=0,07)$ ou duas sementes $(\mathrm{F}=3,13 ; \mathrm{p}=0,08)$ e nem no número total de sementes produzidas $(\mathrm{F}=3,42 ; \mathrm{p}=0,07)$. A estimativa da formação de sementes por indivíduo foi de 105,84 para o morfo brevistilo e 166,74 para o longistilo.
A similaridade do sucesso reprodutivo entre os morfos florais sugere que ambos podem contribuir de maneira semelhante para a manutenção da espécie na área de estudo. Entretanto, estudos realizados com outras espécies distílicas de Psychotria demonstram que o sucesso reprodutivo dos morfos, quando analisados em populações distintas, pode apresentar variações tal como observado em $P$. barbiflora (Monteiro et al. 1991; Teixeira \& Machado 2004a). Nesta espécie, as flores longistilas produziram maior sucesso reprodutivo que as brevistilas, no Cerrado e na Mata Atlântica foram as flores brevistilas. Maior sucesso reprodutivo no morfo brevistilo de $P$. hastisepala também foi observado em sete fragmentos florestais de domínios de Mata Atlântica, no município de Viçosa-MG (Silva 2007).

Nossos resultados explicam parte dos mecanismos de manutenção da espécie na área de estudo, pois demonstram que na população estudada, $P$. carthagenensis apresenta uma razão equilibrada entre os morfos (isopletia). O morfo brevistilo difere do morfo longistilo no comprimento da corola, dos lobos estigmáticos, das anteras e altura do estilete. A espécie apresenta compatibilidade inter e intramorfos e a viabilidade de pólen e os sucessos reprodutivos são semelhantes entre os morfos florais.

\section{Agradecimentos}

A Lucas Eduardo Araújo-Silva, Danilo Souto Ferreira e Felipe Pallis, o incentivo e ajuda na coleta de dados de campo. Aos revisores anônimos, as criticas e sugestões no manuscrito. 
Tabela 4 - Frutos produzidos com uma ou duas sementes e total de sementes produzidas nos morfos florais de Psychotria carthagenensis, em fragmento florestal de mata ciliar, Tangará da Serra - MT.

Table 4-Fruits produced with one or two seeds and produced seeds total in the floral morphs of Psychotria carthagenesis in a riparian forest fragment in Tangará da Serra-MT.

\begin{tabular}{|c|c|c|c|c|c|c|c|c|}
\hline Morfo floral* & $\mathrm{N}^{\circ}$. Ind. & $\mathrm{N}^{\circ}$. Inflor. & $\mathbf{N}^{0}$. Frutos & $\mathbf{P}$ & \multicolumn{2}{|c|}{ Frutos (\%) } & Total sementes & $\mathbf{P}$ \\
\hline & & & & & 1 semente & 2 sementes & & \\
\hline B & 5 & 21 & 1.176 & $0,063 * *$ & 10,9 & 89,1 & 2.224 & $0,072 * *$ \\
\hline $\mathbf{L}$ & 5 & 20 & 1.793 & & 13,1 & 86,9 & 3.351 & \\
\hline
\end{tabular}

\section{Referências Bibliográficas}

Almeida, E.M. \& Alves, M.A. 2000. Fenologia de Psychotria nuda e P. brasiliensis (Rubiaceae) em uma área de Floresta Atlântica no sudeste do Brasil. Acta Botanica Brasilica 14: 335-346.

Andersson, L.A. 1992. A provisional checklist of Neotropical Rubiaceae. Scripta Botanica Belgica 1: $1-119$.

Barrett, S.C.H. 1992. Heterostylous genetic polymorphisms: model systems for evolutionary analysis. In: Barret, S.C.H. (ed.). Evolution and function of heterostyly. Springer-Verlag, Berlim. Pp. 1-29.

Bawa, K.S. \& Beach, J.H. 1983. Evolution of sexual systems in flowering plants. Annals of the Missouri Botanical Garden. 68: 254-274.

Burger, W. \& Taylor, C.M. 1993. Rubiaceae: Flora Costaricensis. Field Museum of Natural History 33: 1-333.

Castro, C.C. \& Oliveira, P.E. 2002. Pollination biology of distylous Rubiaceae in the Atlantic rain forest, SE Brazil. Plant Biology 4: 640-646.

Castro, C.C. \& Araujo, A. C. 2004. Distyly and sequential pollinators of Psychotria nuda (Rubiaceae) in the Atlantic rain forest, Brazil. Plant Systematics and Evolution 244: 131-139.

Castro, C.C.; Oliveira, P.E.A.M. \& Alves, M.C. 2004. Breeding system and floral morphometry of distylous Psychotria L. species in the Atlantic rain forest, SE Brazil. Plant Biology 6: 755-760.

Coelho, C.P. \& Barbosa, A.A.A. 2003. Biologia reprodutiva de Palicourea macrobotrys Ruiz \& Pavon (Rubiaceae): um possível caso de homostilia no gênero Palicourea Aubl. Revista Brasileira de Botânica 26: 403-413.

Coelho, C.P. \& Barbosa, A.A.A. 2004. Biologia reprodutiva de Psychotria poeppigiana Muell. Arg. (Rubiaceae) em mata de galeria. Acta Botanica Brasilica. 18: 481-489.

Consolaro, H.N. 2004. Biologia reprodutiva de duas espécies de Rubiaceae de mata de galeria do Triângulo Mineiro - MG. Dissertação de Mestrado. Universidade Federal de Uberlândia, Uberlândia MG, 58p.
Consolaro, H.; Silva, E.B. \& Oliveira, P.E. 2005. Variação floral e biologia reprodutiva de Manettia cordifolia Mart. (Rubiaceae). Revista Brasileira de Botânica 28: 85-94.

Dafni, A. 1994. Pollination ecology - A practical approach. Oxford University Press. Oxford. 260p.

Dulberger, R. 1992. Floral polymorphisms and their functional significance in the heterostylous syndrome. In: Barret, S.C.H. (ed.). Evolution and function of heterostyly. Springer-Verlag, Berlim. Pp. 41-77.

Ganders, F.R. 1979. The biology of heterostyly. New Zealand Journal of Botany 17: 607-635.

Grandisoli, E.A.C. 1997. Biologia reprodutiva e estrutura da população de Psychotria suterella Müll. Arg. (Rubiaceae) em fragmento de mata secundária em São Paulo (SP). Dissertação de Mestrado. Universidade do Estado de São Paulo, São Paulo. 62p.

Köppen, W. 1948. Climatología. Fondo de Cultura Económica, México. 479p.

Lopes, L.E. \& Buzato, S. 2005. Biologia reprodutiva de Psychotria suterella Muell. Arg. (Rubiaceae) e a abordagem de escalas ecológicas para a fenologia de floração e frutificação. Revista Brasileira de Botânica 28: 785-795.

Lloyd, D.G. \& Schoen, D.J. 1992. Self - and cross fertilization in plants. I. Functional dimensions. International Journal of Plants Sciences 153: 358-369.

Mendonça, L.B. \& Anjos, L. 2006. Flower morphology, nectar features, and hummingbird visitation to Palicourea crocea (Rubiaceae) in the upper Paraná River food plain, Brazil. Annals of the Brazilian Academy of Sciences 78: 45-57

Monteiro, R.; Nakajima, J.N.; Ribeiro, J.L.S. \& Toledo, J.C. 1991. Morfologia e distribuição espacial das formas heterostílicas de Psychotria barbiflora DC. (Rubiaceae). Naturalia 16: 137-146.

Murcia, C. 1996. Forest fragmentation and the pollination of neotropical plants. In: Schellas, J.; Greenberg, R. (eds.). Forest patches in tropical landscapes. Island Press, Washington. Pp.19-36.

Newstron, L.E.; Frankie, G.W. \& Baker, H.G. 1994. A New classification for plant phenology based on flowering patterns in lowland tropical rains forest trees, at La Selva, Costa Rica. Biotropica 26:141-159. 
Passos, L. \& Sazima, M. 1995. Reproductive Biology of the Distylous Manettia luteo-rubra (Rubiaceae). Acta Botanica Brasilica 108: 309-313.

Pereira, Z.V.; Carvalho-Okano, R.M. \& Garcia, F.C.P. 2006a. Rubiaceae Juss. da Reserva Florestal Mata do Paraíso, Viçosa, Minas Gerais, Brasil. Acta Botanica Brasílica 20: 207-224.

Pereira,Z.V.; Vieira, M.F. \& Carvalho-Okano, R.M. 2006 b. Fenologia da floração, morfologia floral e sistema de incompatibilidade em espécies distílicas de Rubiaceae em fragmento florestal do sudeste brasileiro. Revista Brasileira de Botânica 29: 471-480.

Pott, A. \& Pott, V.J. 1994. Plantas do Pantanal. EMBRAPA, Brasília. 320p.

Radford, A.E.; Dickson, W.C.; Massey, J.R. \& Bell, C.R. 1974. Vascular plant systematics. Harper \& Row, New York. 891p.

Ramos, F.N. \& Santos, F.A.M. 2005. Phenology of Psychotria tenuinervis (Rubiaceae) in Atlantic Forest fragments: fragment and habitat scales. Canadian Journal of Botany 83: 1305-1316.

Ribeiro, J.F. \& Walter, B.M.T. 1998. Fitofisionomias do Bioma Cerrado. In: Sano, S.A.; Almeida, S.P. (ed.). Cerrado: ambiente e flora. Embrapa, Planaltina. 556p.

Robbrecht, E. 1988. Tropical woody Rubiaceae. Opera Botanica Belgica 1: 1-127.

Rossi, A.A.B.; Oliveira, L.O. \& Vieira M.F. 2005. Distily and variation in floral traits in natural populations of Psychotria ipecacuanha (Brot.) Stokes (Rubiaceae). Revista Brasileira de Botânica 28: 285-294.
Serigatto, E.M. 2006. Delimitação automática das áreas de preservação permanente e identificação dos conflitos de usos da terra na bacia hidrográfica do Rio Sepotuba-MT. Tese de Doutorado. Universidade Federal de Viçosa, Viçosa. 130p.

Silva, C.A. 2007. Biologia reprodutiva de três espécies distílicas de Psychotria L. e efeitos da fragmentação florestal no sucesso reprodutivo e na diversidade genética de $P$. hastisepala Müll. Arg. (Rubiaceae). Tese de Doutorado. Universidade Federal de Viçosa, Viçosa. 63p.

StatSoft, Inc. 2002. Statistica for Windows (Computer Program Manual). StatSoft Inc., Tulsa.

Taylor, C.M. 1996. Overview of the Psychotrieae (Rubiaceae) in the Neotropics. Opera Botanica Bélgica 7: 261-270.

Teixeira, L.A.G. \& Machado, I.C. 2004a. Biologia da polinização e sistema reprodutivo de Psychotria barbiflora DC. (Rubiaceae). Acta Botanica Brasilica 18: 853-862.

Teixeira, L.A.G. \& Machado, I.C. 2004b. Sabicea cinera Aubl. (Rubiaceae): distilia e polinização em um fragmento de Floresta Atlântica em Pernambuco, Nordeste do Brasil. Revista Brasileira de Botânica 27: 193-204.

Vela, R.H.N.; Dallacort, R. \& Nied, A.H. 2006. Distribuição descendial, mensais e totais de precipitação na região de Tangará da Serra - MT. In: XXXVI Congresso Brasileiro de Engenharia Agrícola. Bonito, MS. 16p. 